\title{
Internalization of Wheat Germ Agglutinin in Endothelial Progenitor Cells in Conventional and Tomographic Electron Microscope Studies
}

\author{
J. Kosiuk, M. Vetterlein, M. Pavelka, J. Neumüller \\ Center for Anatomy and Cell Biology, Dept. of Cell Biology and Ultrastructural Research, Medical \\ University of Vienna, Schwarzspanierstrasse 17, A-1090 Vienna, Austria \\ Internalization of the Wheat Germ Agglutinin (WGA) into the endothelial progenitor cells (EPCs) is \\ a complex process, which is connected with extensive (and significant) membrane dynamics at all \\ stages of endocytosis, including the very first contacts of the WGA with cell surface receptors and \\ the later traffic into the Golgi apparatus (GA). In previous studies with HepG2 hepatoma cells, we \\ showed that WGA-transport into the Golgi apparatus occurs in three steps involving early \\ endosomal compartments, the trans-Golgi network (TGN), which is newly formed during \\ endocytosis, and the stacked Golgi cisternae [1].
}

In our studies, we used cultured CD133-positive cells obtained from human umbilical cord blood. Cells were cultivated on fibronectin (FN)-coated culture vessels in medium 199 containing the cytokines basic fibroblast growth factor (bFGF), epidermal growth factor (EGF) and vascular endothelial growth factor (VEGF). The cultures were pulsed with peroxidase-conjugated WGA (WGA-HRP, $33 \mu \mathrm{g} / 100 \mu \mathrm{l}$ ) for 5 minutes at room temperature and chased at $37^{\circ} \mathrm{C}$ in culture medium without WGA-HRP for various periods of time $(5,10,30,60 \mathrm{~min})$. The WGA-peroxidase was visualized by means of the diaminobenzidine (DAB) reaction. The cells were fixed with glutaraldehyde and reduced $\mathrm{OsO}_{4}$, dehydrated in a graded series of ethanol and embedded in Epon resin. Semi-thin sections of 200-300 nm were performed for electron tomography and $70 \mathrm{~nm}$ ultrathin sections for conventional electron microscopy (EM). Time-dependent progression of uptake and intracellular distribution of WGA were studied in a TECNAI 20 transmission electron microscope with an acceleration voltage of $80 \mathrm{kV}$ for conventional EM and $200 \mathrm{kV}$ for tomography. Digital micrographs of tilt series from $-65^{\circ}$ to $+65^{\circ}$ allow to collect a sufficient amount of data for reconstruction. We used Inspect3D software for reconstruction, and AMIRA software for manufacturing models of the reconstructed cell volumes.

Our studies showed that the WGA-HRP reaction products were attached to the apical side of the membranes, and many of the vesicles were coated. At this early time, coated vesicles also appeared to pinch off from very tiny membrane reticula being localized superficially in the cells. All this changes took place within the first 5 min of an experiment. We observed also a typical discrimination of WGA transport into the GA in three stages. First stage covered the period from 5 to 10 min after application of WGA and was characterized by a high number of coated and notcoated vesicles pinching off from or fussing with large endosomes, which could contain many electron dense vesicles accumulated in their lumen (Fig. 1). At that time, the trans Golgi network (TGN) was reduced and not visible. In the period of 10 to 30 min of WGA internalization, the new formation of the transport organelles was observed. The vesicles appeared to accumulate in the region of the TGN in vicinity of the Golgi apparatus (Fig. 2) The second stage was dominated by the reorganization of the TGN region: The TGN extended and appeared with the large endosomes. In TGN architecture, tubular and vesicular components were present, both filled with reactions products. At this point of time the TGN attains a maximum of complexity and up then became reduced in size. The last part of the internalization took place between 30 to $60 \mathrm{~min}$ of the 
experiment. At this stage, single spots of WGA reactions products were visible within the Golgi cisternae.

As our data showed, that the internalization of WGA-HRP is a highly dynamic event involving almost all membrane compartments in the EPCs

In current studies, the routes of endocytosis are of particular interest in endothelial progenitor cells at early maturation stages characterized by the occurrence of early forms of Weibel-Palade bodies [2]

\section{References:}

[1] Vetterlein M, Ellinger A, Neumuller J, Pavelka M. Histochem Cell Biol. 117(2) (2002) 143-50 [2] Neumüller J, Vetterlein M, Neumüller-Guber SE, Kosiuk J, Huber J, Pavelka M. Microscopy Conference 2005, 6. Dreiländertagung, Davos, Switzerland, August 28 - September 2, 2005

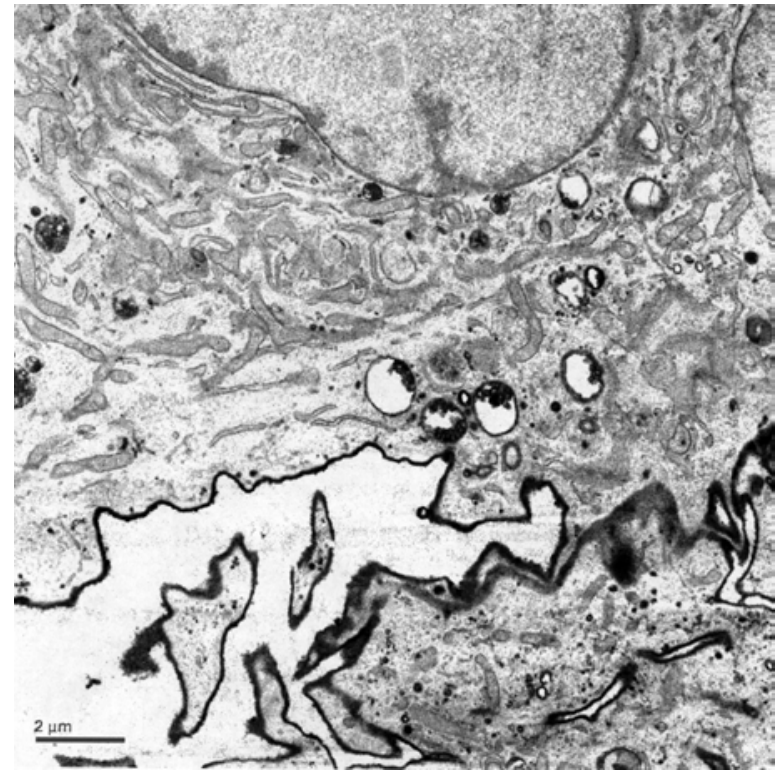

Fig. 1. Saw-like protrusions of the cell surface. Membrane covered with layer of electron dense reactions products. Large number of vesicles is visible under the cell membrane.

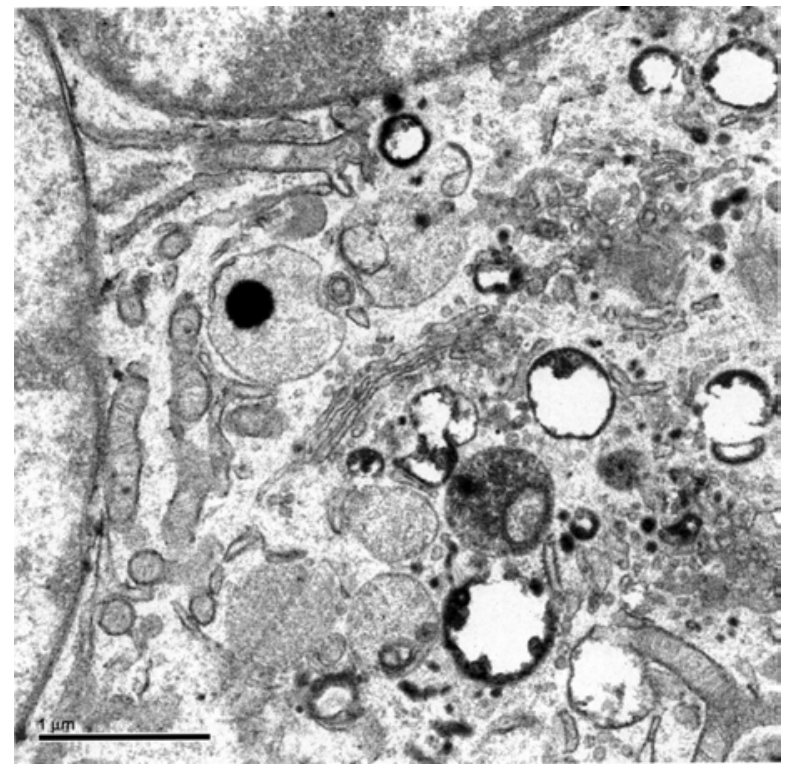

Fig. 2. Golgi vicinity is full of vesicles containing small endosomes with reactions products. Major part of the vesicles is located at the TGN side of the GA. 